\title{
Ein theoretisches Modell zur Integration von Arbeitsmigranten in Organisationen
}

\author{
Julia A. M. Reif ${ }^{1}$ Erika Spieß ${ }^{1} \cdot$ Rita Berger $^{2}$
}

(C) Springer Fachmedien Wiesbaden 2017

Zusammenfassung In diesem Beitrag der Zeitschrift „Gruppe. Organisation. Interaktion. (GIO)“ wird ein Modell organisationaler Akkulturation entwickelt, das beschreibt, wie beim Eintritt von Arbeitsmigrant/-innen in Organisationen durch das Zusammentreffen unterschiedlicher Kulturen akkulturative und sozialisationsbedingte Stressoren entstehen und das dabei auch die Perspektive der bereits in der Organisation tätigen Personen berücksichtigt. Dabei werden Ansätze der Akkulturationsforschung mit Ansätzen der Sozialisationsforschung verknüpft. Intrapersonelle (zyklisches Zusammenspiel von Information, Unsicherheitsreduktion und Lernen) und interpersonelle (Voice, Partizipation, Empowerment, soziale Unterstützung) Copingprozesse führen zu einer gelungenen Anpassung der Arbeitsmigrant/-innen. Eine erfolgreiche Anpassung wirkt sich wiederum positiv auf weitere arbeitsbezogene Ergebnisse aus. Das Modell berücksichtigt, dass durch die zusätzliche Herausforderung der Anpassung an eine neue Kultur die organisationale Sozialisation für Arbeitsmigrant/-innen komplexer verläuft. Das Modell liefert Ansatzpunkte für Forschung und Praxis.

Schlüsselwörter Migration · Arbeitsmigration · Akkulturation · Organisationale Sozialisation - Stress · Coping

Dr. Julia A. M. Reif

Julia.reif@psy.lmu.de

1 Department Psychologie, Lehrstuhl Wirtschafts- und Organisationspsychologie, Ludwig-Maximilians-Universität München, Leopoldstraße 13, 80802 München, Deutschland

2 Fakultät für Psychologie, Abteilung für Sozialpsychologie und für Quantitative Psychologie, University of Barcelona, Passeig de la Vall d'Hebron, 171, E-08035 Barcelona, Spanien

\section{A theoretical model of the integration of migrant workers in organizations}

Abstract This article in the journal "Gruppe. Organisation. Interaktion. (GIO)" portraits a model of organizational acculturation that describes the emergence of acculturative and socialization stressors upon the encounter of different cultures when migrant workers enter organizations, while taking into account the perspective of persons already employed in those organizations. This model combines approaches of acculturation and socialization research. Intrapersonal (cyclic interplay of information, uncertainty reduction and learning) and interpersonal (voice, participation, empowerment and social support) coping-processes lead to successful adaptation of work migrants. In turn, successful adaptation has positive effects on further work-related outcomes. The model takes account of the fact that due to the additional challenge of having to adapt to a new culture, organizational socialization of work migrants proceeds in a more complex fashion. The model provides starting points for future research and practical applications.

Keywords Migration - Work-related migration · Acculturation - Organizational socialization · Stress · Coping

\section{Einleitung}

Deutschland ist ein Einwanderungsland (Angela Merkel, im Bürgerdialog ${ }^{1}$ ). Die sog. „Flüchtlingskrise“ in 2015 (Wiesendahl 2016) hat die Frage der Integration von Arbeits-

\footnotetext{
1 Vgl. http://www.faz.net/aktuell/politik/ausland/europa/angela-merkelsieht-deutschland-als-einwanderungsland-13623846.html.
} 


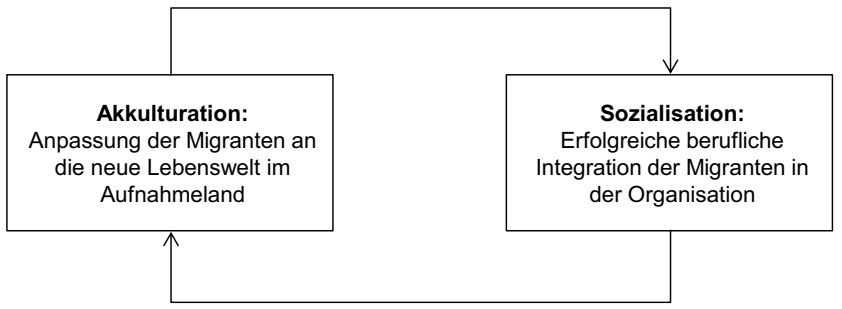

Abb. 1 Wechselseitige Beeinflussung von Akkulturation und Sozialisation

migranten erneut aktuell werden lassen. Der anhaltende Flüchtlingsstrom zeigt, dass Migration und Integration zentrale Zukunftsthemen der Politik sowie in der öffentlichen Diskussion sind (Bundesamt für Migration und Flüchtlinge 2016). Bei der Integration von Migrant/-innen stehen die Anpassung an die neue Lebenswelt im Aufnahmeland (Akkulturation) und die erfolgreiche berufliche Integration (Sozialisation in der Organisation) in engem Zusammenhang. Dabei leistet einerseits die Akkulturation im Aufnahmeland einen wichtigen Beitrag zum beruflichen Erfolg der Migrant/-innen. Andererseits kann eine berufliche Beschäftigung der Migrant/-innen zur erfolgreichen Akkulturation beitragen (Ward und Geeraert 2016; Fleay et al. 2013), denn auch für Flüchtlinge besteht die Bedeutung von Arbeit in einer neuen Lebensperspektive, sozialen Kontakten, Wertschätzung in der Gesellschaft und einem neuen Selbstwertgefühl (Abb. 1).

Aus wissenschaftlicher Sicht beschäftigt sich u.a. die interkulturelle Psychologie (Berry 1997; Ward 1996) mit der Anpassung an fremde Kulturen. Mit der Frage der Integration in den Arbeitsmarkt und in Organisationen sind vor allem Soziologie und Organisationspsychologie befasst. Für die organisationale Sozialisation wiederum wurde bislang jedoch der kulturelle Aspekt bzw. der Migrationshintergrund kaum berücksichtigt (vgl. Ashforth et al. 2007; für eine Ausnahme siehe Malik et al. 2014). Ziel des vorliegenden Beitrages ist es, diese beiden Ansätze - die Anpassung an fremde Kulturen und die Sozialisation in Organisationen - in einem Modell organisationaler Akkulturation miteinander zu verbinden.

\subsection{Akkulturation}

Berry (1997) und Ward (1996) definieren Akkulturation, also die „Anpassung an eine fremde Kultur“ als Hineinwachsen einer Person in ihre kulturelle Umwelt. Berry (1997) unterscheidet vier Akkulturationsstrategien: Integration (starke Orientierung an der Kultur des Aufenthalts- und des Herkunftslandes), Assimilation (stärkere Orientierung an der Kultur des Aufenthaltslandes), Separation (stärkere Orientierung an der Kultur des Herkunftslandes) und Marginalisierung (schwache Orientierung beiden Kulturen gegen- über). In Abhängigkeit von diesen Strategien (und weiteren Faktoren) werden Akkulturationserfahrungen unterschiedlich wahrgenommen und bewältigt, wovon kurzfristige Stressreaktionen und langfristig der Anpassungserfolg abhängen.

Ward (1996) unterscheidet zwischen psychologischer und soziokultureller Anpassung: Ausgehend von einem kulturellen Kontakt zwischen der Herkunftskultur und der Kultur im Aufnahmeland kommt es zu einer Stressreaktion, die sich wiederum auf Emotionen, Verhalten und Kognitionen auswirkt. Die Stärke dieser Auswirkung wird moderiert durch Charakteristika der Situation (z. B. kulturelle Distanz, Stärke des Kontakts) und Charakteristika der Person (z. B. Beherrschung der neuen Sprache, Persönlichkeit). Ergebnis dieses Prozesses ist psychologische und soziokulturelle Anpassung. Ward und Geeraert (2016) erweitern diesen Grundgedanken um Faktoren wie kulturelle Bewusstheit und betten ihn in einen größeren Kontext von Familie, Institutionen und Organisationen, sowie der Gesellschaft ein.

Safdar et al. (2003) entwickelten das sog. MIDA-Modell, das den individuellen Unterschieden im Akkulturationsprozess Rechnung tragen soll (siehe auch Safdar et al. 2009). In diesem Modell werden drei der Hauptziele von Immigranten in multikulturellen Gesellschaften untersucht: die Beibehaltung der eigenen Kultur, die Teilnahme an der neuen Gesellschaft und - als ein neuer Aspekt - die Wahrung psychischer und physischer Gesundheit.

Alle diese Modelle beziehen sich jedoch nicht auf die Situation von Migrant/-innen in Organisationen bzw. den organisationalen Sozialisationsprozess. Dieser ist jedoch von großer Bedeutung für die Integration von Arbeitsmigrant/ -innen wie auch von Flüchtlingen.

\subsection{Sozialisation}

Organisationale Sozialisation umfasst alle Prozesse der Integration neuer Mitarbeiter/-innen in die Organisation (Wihler et al. 2014). Die ersten Monate in der Organisation können eine weitreichende Konsequenz für die spätere Bindung an die Organisation und die gesamte berufliche Entwicklung haben (Spieß und von Rosenstiel 2010). Oft wird organisationale Sozialisation in Stufen- oder Phasenmodellen beschrieben (Saks und Ashforth 1997; Ashforth et al. 2007). Von Seiten des Unternehmens werden häufig Sozialisationstaktiken, z. B. spezielle Einführungstrainings, eingesetzt (Jones 1986), um die Informationen zu gestalten, die Neueinsteiger/-innen erhalten (van Maanen und Schein 1979, siehe auch Saks et al. 2007).

Neueinsteiger/-innen können aber auch selbst durch proaktive Verhaltensweisen in den Prozess der eigenen Sozialisation miteinbezogen werden (Saks und Gruman 2011; Griffin et al. 2000; Kammeyer-Mueller und Wan- 
Abb. 2 Modell organisationaler Akkulturation

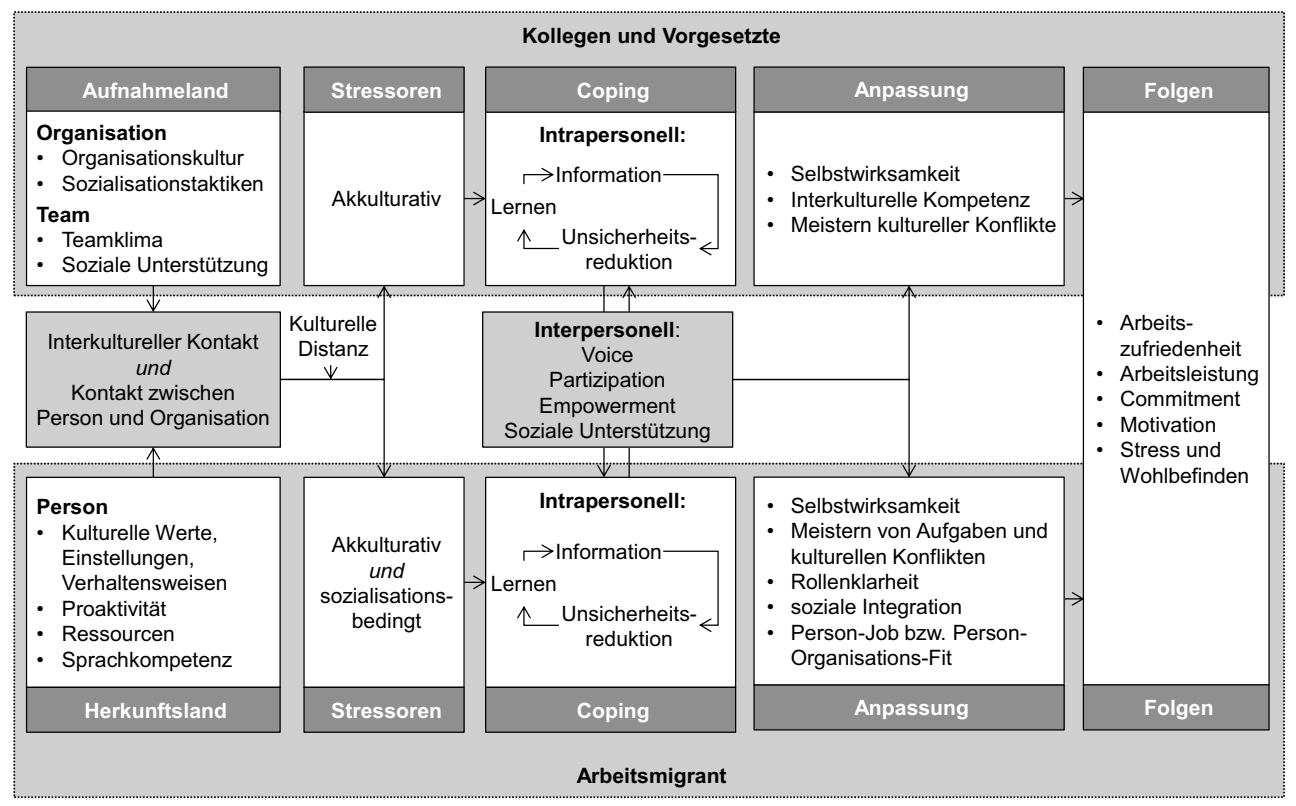

berg 2003). Proaktive Verhaltensweisen beziehen sich vor allem auf das aktive Einholen von Informationen (siehe auch Bauer et al. 2007). Das Verfügen über Informationen kann Unsicherheit reduzieren (Miller und Jablin 1991), sowie Selbstwirksamkeit (Wood und Bandura 1989) und Lernen fördern. Lernen wird häufig als das „Herzstück“ aller Sozialisationsmodelle beschrieben (Ashforth et al. 2007, S. 16), da Neueinsteiger/-innen für eine erfolgreiche Integration Normen, Werte, Aufgaben und Rollen lernen müssen, die die Organisation und ihre Teams kennzeichnen (Cooper-Thomas und Anderson 2006). Lernen steht in engem Zusammenhang mit dem zur Verfügung stehenden „sozialen Kapital“, also dem sozialen Netzwerk in der Organisation, auf das im Lernprozess zurückgegriffen werden kann (Fang et al. 2011).

\section{Ein Modell organisationaler Akkulturation}

Ausgehend von dem Grundgedanken, dass Arbeitsmigrant/innen vor multiplen Herausforderungen stehen - der Akkulturation in einem neuen Land und der Sozialisation in einer neuen Organisation (siehe auch Ferris et al. 2014; Malik et al. 2014) - beschreibt das Modell der organisationalen Akkulturation (Abb. 2) den Prozess der Integration von Arbeitsmigrant/-innen in Organisationen, ausgehend von organisationalen, relationalen und individuellen Antezedenzien. Die Organisation, sowie die Teams innerhalb der Organisation befinden sich in der Kultur des Aufnahmelandes, während die Arbeitsmigrant/-innen als Individuen unter dem Einfluss ihrer Herkunftskultur stehen.

\subsection{Die Organisation}

Die Organisation ist von ihrer Organisationskultur geprägt, wovon wiederum abhängt, welche Werte in der Organisation von zentraler Bedeutung sind. Die Organisationskultur zeigt sich in beobachtbaren Verhaltensregelmäßigkeiten, dominanten Werten oder Symbolen und spielt für die Sozialisation neuer Mitglieder eine große Rolle (Schein 1985). Organisationen verfolgen häufig bestimmte Sozialisationstaktiken, also etwa Einstiegs- oder Sozialisationsprogramme für neue Mitarbeiter/-innen, die sich gemäß verschiedener Metaanalysen positiv auf die Integration auswirken (Bauer et al. 2007; Saks et al. 2007).

\subsection{Das Team}

Da Sozialisation ein Prozess ist, der innerhalb des Teamkontextes stattfindet (Major et al. 1995), wird die Integration von Arbeitsmigrant/-innen vom Teamklima und, damit einhergehend, dem Ausmaß an sozialer Unterstützung im Team beeinflusst (z. B. Ryan et al. 2008). Aufgrund der Tatsache, dass Immigrant/-innen einer Vielzahl von Stressoren ausgesetzt sind wie z. B. der Erfahrung, in (mit) zwei Kulturen leben zu müssen und zu einem gewissen Grad an beiden teilzuhaben, Sprach- bzw. Kommunikationsschwierigkeiten, Diskriminierung, Heimweh, oder Einsamkeit (siehe z. B. Berry 1997; Ward et al. 2001), ist sozioemotionale Unterstützung durch Familie, Freund/-innen oder aber auch durch Kontakte mit Einheimischen (also z. B. Kolleg/-innen) von besonderer Bedeutung (Uslucan 2013). So zeigte sich in einer Studie von Podsiadlowski et al. (2012), dass der Kontakt mit den Einheimischen eine wichtige Bedin- 
gung bei einer erfolgreichen Anpassung von Expatriates darstellte.

\subsection{Die Person}

Menschen eignen sich in ihrer Herkunftskultur individuelle Werte, Einstellungen und Verhaltensweisen an, die (auch im Aufnahmeland) als eine Orientierung für Denken und Handeln herangezogen werden (Ng et al. 2016; Schwartz 2011). Kulturen unterscheiden sich z. B. darin, wie Menschen mit Unsicherheit und Risiko umgehen, welche Rolle Tradition spielt, welche Unterschiede zwischen Männern und Frauen betont werden, und wie mit Machtgefälle umgegangen wird (Hofstede und Hofstede 2005). Aus diesen unterschiedlichen Werthaltungen erwachsen unterschiedliche Präferenzen und Arbeitseinstellungen. Für Personen aus kollektivistischen Kulturen haben z. B. Werte wie Familie und Harmonie einen hohen Stellenwert; bei Personen aus individualistisch geprägten Kulturen steht oft der eigene Erfolg im Zentrum (Ringeisen 2013).

Menschen unterscheiden sich auch in ihrer Proaktivität, also dem Ausmaß, in dem sie eigeninitiativ und vorausschauend Einfluss auf sich und ihre Umwelt nehmen (Tornau und Frese 2013; siehe auch Stroppa und Spieß 2011). Für einen erfolgreichen Integrationsprozess ist es wichtig, dass der oder die Einzelne aktiv und selbständig seine Beziehungen am neuen Arbeitsplatz gestaltet (Saks und Gruman 2011; Reif und Brodbeck 2014, 2017). Durch proaktive Verhaltensweisen gelangen Neueinsteiger/-innen an mehr und nützlichere Information (Comer 1991). Auch im Bereich der Akkulturationsforschung wird auf das Konzept der Proaktivität hingewiesen (Richmond 1993).

Das Vorhandensein persönlicher Ressourcen (vgl. auch Ellis et al. 2015) familiärer oder sozialer Art (z. B. Netzwerke), sowie die Sprachkompetenz werden im Prozess organisationaler Akkulturation zudem als wichtige persönliche Einflussfaktoren betrachtet (Lehner und Spieß 2013).

\subsection{Akkulturative Stressoren}

Beim Eintritt von Arbeitsmigrant/-innen in eine neue Organisation entsteht Kontakt zwischen der Kultur des Herkunftslandes der Arbeitsmigrant/-innen und der Kultur der Organisation im Aufnahmeland. Dieser Kontakt löst akkulturative Stressoren aus, wobei die Stärke der Stressoren von der Höhe der kulturellen Distanz zwischen Herkunfts- und Aufnahmeland abhängt: je höher die Distanz, desto stärker die Stressoren. Unter kultureller Distanz (Church 1982) versteht man das Ausmaß, in welchem sich Gruppen kulturell unterscheiden.

Akkulturative Stressoren betreffen nicht nur die Arbeitsmigrant/-innen, sondern auch die bereits in der Organisation arbeitenden Personen. Akkulturative Stressoren, die sowohl
Arbeitsmigrant/-innen als auch Kolleg/-innen und Vorgesetzte betreffen können, sind beispielsweise ein Gefühl der Bedrohung der eigenen kulturellen Identität, Angst und Unsicherheit (Angst, etwas falsch zu machen, Unsicherheit darüber, wie man sich verhalten soll), Ärger und Enttäuschung (z.B. durch die Konfrontation mit stereotypen Äußerungen oder rassistischen Witzen), Misstrauen und Kommunikationsprobleme. Durch Kommunikationsprobleme können sich für Arbeitsmigrant/-innen Verständnisschwierigkeiten bei Arbeitsaufgaben ergeben, die sie dadurch nicht ordnungsgemäß erledigen können. Kolleg/-innen und Vorgesetzte müssen dann Zeit und Geduld darauf verwenden, den Neulingen die Arbeitsschritte zu erklären. Hinzu können für die Arbeitsmigrant/-innen noch Stressoren wie wahrgenommene Diskriminierung (fehlender Zugang zu bestimmten Möglichkeiten, Gefühl der ungleichen Behandlung), soziale Isolierung, Heimweh oder Minderwertigkeitsgefühle kommen (Sandhu und Asrabadi 1994).

Welche Vorkommnisse oder Gegebenheiten im Arbeitskontext nun tatsächlich als Stressoren wahrgenommen werden hängt wiederum von der Kultur der Betroffenen ab: In Ländern mit einer hohen Ausprägung von Kollektivismus werden z.B. zwischenmenschliche Konflikte und mangelnde Klarheit als besonders belastend erlebt, da sie die relevanten kulturellen Werte - z. B. harmonische Beziehungen - bedrohen.

\subsection{Sozialisationsbedingte Stressoren}

Migrant/-innen stehen beim Eintritt in eine neue Organisation nicht nur vor der Herausforderung der Bewältigung von akkulturativen Stressoren, sondern auch vor der Herausforderung der Bewältigung sozialisationsbedingter Stressoren. Diese sozialisationsbedingten Stressoren (Rollenkonflikt, Rollenambiguität, Rollenüberlastung, nicht erfüllte Erwartungen) entstehen, wenn Personen neu in eine Organisation einsteigen (Saks und Ashforth 2000).

Sowohl in Akkulturationsmodellen (vgl. Ward und Geeraert 2016), als auch in Modellen organisationaler Sozialisation (vgl. Ellis et al. 2015) wird - aber unabhängig voneinander - ein Bezug zu Stress hergestellt und auf Stresstheorien zurückgegriffen. Eine stress- und coping-fokussierte Herangehensweise an das Thema organisationaler Akkulturation bietet daher einen Ansatzpunkt zur Verknüpfung dieser beiden Forschungstraditionen.

\subsection{Coping}

Erfolgreiche organisationale Akkulturation hängt von der Bewältigung dieser akkulturativen und sozialisationsbedingten Stressoren ab, sowohl auf Seiten der Arbeitsmigrant/-innen als auch auf Seiten der in der Organisation bereits tätigen Personen, also Kolleg/-innen und Vorgesetz- 
te. Coping wird oft in Zusammenhang mit Akkulturation beschrieben (Ward und Geeraert 2016), ist aber auch hinsichtlich des Zurechtkommens beim Eintritt in eine neue Organisation von Relevanz (Louis 1980).

Zentrale psychologische Mechanismen des Copings (sowohl auf Seiten von Arbeitsmigrant/-innen als auch auf Seiten von Kolleg/-innen und Vorgesetzten) sind hierbei Unsicherheitsreduktion und Lernen, die einen Kreislauf mit dem Bereitstellen und Aufnehmen von Informationen bilden: Durch das Aufnehmen bereitgestellter Informationen kann Unsicherheit reduziert werden, was wiederum Lernen und das Suchen neuer Informationen fördert. Diese Informationen sollten sich nicht nur auf aufgaben- oder organisationsspezifische Aspekte beziehen, sondern auch kulturelles Wissen beinhalten, wie etwa kulturelle, geschichtliche und politische Hintergründe zum Herkunftsland von Arbeitsmigranten, aber auch zum Aufnahmeland. Auch sollten hier Informationen zum Umgang mit kulturellen Konflikten gegeben werden. Durch die Reduktion von Unsicherheit wird die Arbeitsumgebung vorhersehbarer, verständlicher und kontrollierbarer (Saks und Ashforth 1997). Coping sollte aber nicht isoliert in zwei „Lagern“ stattfinden.

Um die Brücke zwischen Arbeitsmigrant/-innen einerseits und Kolleg/-innen und Vorgesetzten andererseits zu schlagen, bedarf es eines gegenseitigen Austausches (siehe auch Cooper-Thomas und Anderson 2006). Empowerment und soziale Unterstützung durch Kolleg/-innen und Vorgesetzte sollen Arbeitsmigrant/-innen persönliche Kontrolle ermöglichen und den Prozess des Lernens unterstützen. Arbeitsmigrant/-innen wird dadurch Partizipation ermöglicht und eine „Stimme“ gegeben (vgl. das im englischsprachigen als „Voice“ bezeichnete psychologische Konstrukt). Diese Faktoren sollten sich positiv auf den Coping-Prozess auswirken, da sie den Arbeitsmigrant/-innen Autonomie ermöglichen (siehe auch Taormina 2009).

\subsection{Anpassung}

Eine erfolgreiche Bewältigung von akkulturativen und sozialisationsbedingten Stressoren sollte eine erfolgreiche Anpassung der Arbeitsmigrant/-innen begünstigen, die gekennzeichnet ist von Selbstwirksamkeit (Saks und Gruman 2011; Bauer et al. 2007; Wood und Bandura 1989), dem Aneignen von Fähigkeiten und dem Meistern von Arbeitsaufgaben (Saks und Ashforth 1997; Choi 2014) und kulturellen Konflikten (Malik et al. 2014), Rollenklarheit (Saks und Ashforth 1997; Bauer et al. 2007), sozialer Integration (Saks und Ashforth 1997) bzw. sozialer Akzep$\operatorname{tanz}$ (Bauer et al. 2007), sowie Person-Job bzw. PersonOrganisations-Fit (Saks und Ashforth 1997).

\subsection{Folgen}

Eine erfolgreiche Anpassung sollte sich auf Arbeitszufriedenheit, Arbeitsleistung, Commitment, Motivation, sowie Stress und Wohlbefinden auswirken. Diese Zusammenhänge wurden in bestehender Forschung zu Akkulturation und Sozialisation bereits angenommen und gezeigt (siehe beispielsweise Saks und Gruman 2011, 2014; Bauer et al. 2007; Ellis et al. 2015). Der bzw. die Einzelne erbringt gute Leistung, ist zufrieden und der Organisation verbunden, die Organisation wiederum verfügt über motivierte Mitarbeitende, die zum wirtschaftlichen Erfolg beitragen.

\section{Diskussion}

Das Modell organisationaler Akkulturation integriert Ansätze aus den Forschungstraditionen der Akkulturation und Sozialisation. Organisationale Kultur, Sozialisationstaktiken, Teamklima und soziale Unterstützung durch das Arbeitsteam, individuelle Ressourcen, Einstellungen, Werte und Verhaltensweisen, sowie Proaktivität werden beim Zusammentreffen unterschiedlicher Kulturen im organisationalen Sozialisationsprozess als Prädiktoren für das Entstehen akkulturativer und sozialisationsbedingter Stressoren angenommen. Eine Bewältigung der Stressoren durch intrapersonelle Prozesse der Informationsaufnahme, Unsicherheitsreduktion und des Lernens, sowie ein interpersoneller Austausch in Form von sozialer Unterstützung, Empowerment, Partizipation und dem „Geben einer Stimme“ (Voice) führt zu einer gelungenen Anpassung, die sich in sozialer Integration, Rollenklarheit, dem Meistern von Arbeitsaufgaben und kulturellen Konflikten und einem Person-Job bzw. Person-Organisations-Fit äußert. Eine erfolgreiche Anpassung wirkt sich wiederum positiv auf weitere arbeitsbezogene Faktoren aus.

\subsection{Implikationen für die Forschung}

Zukünftige Forschung sollte aus dem Modell Moderationsund Mediationshypothesen ableiten, die mittels längsschnittlicher Studien überprüft werden könnten. So könnte beispielsweise die relative Bedeutung von organisationsbezogenen, teambezogenen und persönlichen Antezedentien auf das Entstehen akkulturativen und sozialisationsbedingten Stresses untersucht werden, in Abhängigkeit von der kulturellen Distanz. Des weiteren sollte untersucht werden, inwiefern sich interpersonelle Copingprozesse (Voice, Partizipation, soziale Unterstützung, Empowerment) auf intrapersonelle Copingprozesse auswirken und wie diese letztlich die Anpassung beeinflussen. Zukünftige Forschung sollte zudem differenziert untersuchen, welche Art an Informationen auf welchen Ebenen von un- 
terschiedlichen Personengruppen benötigt werden, um den intrapersonellen Lernprozess in Gang zu setzen.

\subsection{Implikationen für die Praxis}

Das Modell organisationaler Akkulturation liefert Ansatzpunkte für organisationale Interventionen, die sich sowohl auf Arbeitsmigrant/-innen als auch auf die mit ihnen interagierenden Personen beziehen. Insbesondere Maßnahmen, die den Prozess des Copings unterstützen, sollten eine organisationale Akkulturation positiv beeinflussen.

Der zirkuläre intrapersonelle Copingprozess, bestehend aus Information, Unsicherheitsreduktion und Lernen könnte z.B. durch gezielte Informationsprogramme getriggert werden. Praktiker/-innen könnten hierzu Arbeitsmigrant/ -innen Zugang zu verschiedenen Informationsquellen bieten: Kolleg/-innen, Vorgesetzte und Mentor/-innen sind die hilfreichsten Quellen, um die wichtigsten „Spielregeln“ der Organisation zu lernen (siehe Cooper-Thomas und Anderson 2006). Oft reicht ein Mentor oder eine Mentorin nicht aus (Ferris et al. 2014), weshalb mehrere Mentor/-innen verfügbar sein sollten, z. B. für emotionale, aufgabenbezogene, technische und karrierebezogene Unterstützung, und für Unterstützung hinsichtlich des Verstehens organisationaler Politik (Allen et al. 1999). Auch allgemeine Unterlagen für Neueinsteiger/-innen sowie weitere Unternehmensliteratur können Informationen liefern. Durch diese Quellen könnten Arbeitsmigrant/-innen, aber auch Kolleg/-innen und Vorgesetzte Wissen in verschiedenen Domänen erwerben (vgl. Cooper-Thomas und Anderson 2006; Chao et al. 1994):

- Aufgaben- und Rollen: Was ist meine Aufgabe? Welche Rolle nehme ich ein? Welche Verantwortlichkeiten nehme ich wahr? Wo sind relevante Schnittstellen? Woran wird meine Leistung gemessen?

- Soziales: Wer sind meine Kollegen? In welches Team bin ich eingebunden? Wer kann mit wem?

- Kultur, Werte und Sprache der Organisation: Wofür steht die Organisation? Welche impliziten Verhaltensregeln gibt es? Welchen Stellenwert nimmt Hierarchie ein? Welches typische Vokabular wird verwendet?

- Unternehmenspolitik: Wie laufen die Dinge „wirklich“ ab? Wer sind die einflussreichen Personen? Wer hat was zu sagen? Was sind die Hintergründe bestimmter Entscheidungen oder Handlungen?

- Kultur und interkultureller Kontakt: Welche Werte, Einstellungen und Verhaltensweisen sind mit bestimmten Kulturen verbunden? Wie kann sich das auf Verhalten im Arbeitskontext übertragen? Was führt in verschiedenen Kulturen zu Stress? Wie wird in verschiedenen Kulturen mit Stress umgegangen?

Begleitend könnten Trainings zur Förderung persönlicher Initiative eingesetzt werden. Praktiker/-innen könnten
Neueinsteiger/-innen ermuntern, aktiv Informationen und Rückmeldung einzuholen, Beziehungen zu knüpfen und eigene Rollen und Aufgaben zu „hinterfragen“ (siehe auch Griffin et al. 2000).

Hinsichtlich interpersoneller Copingprozesse könnten Coachings zum Thema „Voice“ für Arbeitsmigrant/-innen angeboten werden, in denen vermittelt wird, dass bzw. an welchen Stellen und auf welche Weise die eigene Meinung eingebracht werden kann. Auch Partizipationsmöglichkeiten und -techniken könnten in diesem Zusammenhang vermittelt werden. Dazu muss auf Team- und Führungsebene für ein Klima der Offenheit und der psychologischen Sicherheit gesorgt werden, welches das freie Einbringen eigener Meinungen, Ideen und Vorschläge zulässt. Kolleg/ -innen und Vorgesetzte hinsichtlich ihrer Fähigkeit zu fördern, Verantwortung und Macht an Arbeitsmigrant/-innen abzugeben und Handlungsspielräume zu betonen (Empowerment), wäre ein weiterer Ansatzpunkt. Auch könnten gezielt Techniken der sozialen Unterstützung, begleitet durch vertrauensbildende Maßnahmen, vermittelt werden.

\section{Fazit}

Organisationale Akkulturation ist ein interaktiver Prozess, da sich Arbeitsmigrant/-innen und die Mitglieder der Organisation in einem wechselseitigen Austausch befinden. Dieser Austauschprozess kann von der Organisation aktiv gesteuert werden. Um den Prozess organisationaler Akkulturation in eine erfolgreiche Integration münden zu lassen, sollten beide Seiten bemüht sein, ein gegenseitiges Verständnis für kulturelle Unterschiede aufzubringen. Das würde im Sinne von Bennett (1998) bedeuten, kulturelle Unterschiede nicht zu leugnen, sondern diese anzuerkennen und lernen mit ihnen umzugehen.

\section{Literatur}

Allen, T.D., McManus, S.E., \& Russell, J.E. (1999). Newcomer socialization and stress: Formal peer relationships as a source of support. Journal of Vocational Behavior, 54(3), 453-470.

Ashforth, B.E., Sluss, D. M., \& Harrison, S.H. (2007). Socialization in organizational contexts. In G.P. Hodgkinson, J. K. Ford, G. P. Hodgkinson \& J. K. Ford (Hrsg.), International review of industrial and organizational psychology 2007, (Bd. 22, S. 1-70). New York: John Wiley \& Sons Ltd.

Bauer, T.N., Bodner, T., Erdogan, B., Truxillo, D. M., \& Tucker, J. S (2007). Newcomer adjustment during organizational socialization: A meta-analytic review of antecedents, outcomes, and methods. Journal of Applied Psychology, 92(3), 707-721. doi:10. 1037/0021-9010.92.3.707.

Bennett, M. J. (1998). Intercultural communication: a current perspective. In M. Bennett (Hrsg.), Basic concepts of intercultural communication: selected readings (S. 1-34). Yarmouth: Intercultural Press.

Berry, J. W. (1997). Immigration, acculturation and adaptation. Applied Psychology: An International Review, 46, 5-34. 
Bundesamt für Migration und Flüchtlinge (BAMF), \& Bundesministerium des Innern (BMI) (2016). Migrationsbericht des Bundesamtes für Migration und Flüchtlinge im Auftrag der Bundesregierung. Berlin: Bundesministerium des Innern.

Chao, G.T., O’Leary-Kelly, A. M., Wolf, S., Klein, H. J., \& Gardner, P.D. (1994). Organizational socialization: Its content and consequences. Journal of Applied psychology, 79(5), 730.

Choi, Y. (2014). Newcomer socialization: the roles of social networks. Doctoral dissertation. Minneapolis: University of Minnesota.

Church, A. (1982). Sojourner adjustment. Psychological Bulletin, 9 , $540-572$.

Comer, D. R. (1991). Organizational newcomers' acquisition of information from peers. Management communication quarterly, 5(1), $64-89$.

Cooper-Thomas, H. D., \& Anderson, N. (2006). Organizational socialization: A new theoretical model and recommendations for future research and HRM practices in organizations. Journal of Managerial Psychology, 21(5), 492-516.

Ellis, A.M., Bauer, T.N., Mansfield, L.R., Erdogan, B., Truxillo, D. M., \& Simon, L. S. (2015). Navigating uncharted waters: Newcomer socialization through the lens of stress theory. Journal of Management, 41(1), 203-235. doi:10.1177/0149206314557525.

Fang, R., Duffy, M.K., \& Shaw, J.D. (2011). The organizational socialization process: Review and development of a social capital model. Journal of Management, 37(1), 127-152.

Ferris, G.R., Daniels, S.R., \& Sexton, J.C. (2014). Race, stress, and well-being in organizations: An integrative conceptualization. In P. L. Perrewé, C. C. Rosen \& J. R. B. Halbesleben (Hrsg.), The role of demographics in occupational stress and well being (S. 1-39). Bingley, UK: Emerald Group Publishing Limited.

Fleay, C., Hartley, L., \& Kenny, M. A. (2013). Refugees and asylum seekers living in the Australian community: the importance of work rights and employment support. Australian Journal of Social Issues, 48(4), 473-493.

Griffin, A. E., Colella, A., \& Goparaju, S. (2000). Newcomer and organizational socialization tactics: An interactionist perspective. $\mathrm{Hu}$ man Resource Management Review, 10(4), 453-474.

Hofstede, G., \& Hofstede, G.J. (2005). Cultures and organizations: Software of the mind, revised and expanded (2. Aufl.). New York: McGraw-Hill.

Jones, G.R. (1986). Socialization tactics, self-efficacy, and newcomers' adjusment to organizations. Academy of Management Journal, 29, 262-279.

Kammeyer-Mueller, J.D., \& Wanberg, C.R. (2003). Unwrapping the organizational entry process: disentangling multiple antecedents and their pathways to adjustment. Journal of Applied Psychology, $88(5), 779$.

Lehner, M., \& Spieß, E. (2013). Systematische Integration hochqualifizierter Arbeitsmigranten. Personalführung, 1, 22-29.

Louis, M.R. (1980). Surprise and sense making: what newcomers experience in entering unfamiliar organizational settings. Administrative Science Quarterly, 64, 226-251.

Van Maanen, J., \& Schein, E.H. (1979). Toward a theory of organizational socialization. In B. M. Staw (Hrsg.), Research in organizational behavior (Bd. 1, S. 209-264). Greenwich CT: JAI Press.

Major, D. A., Kozlowski, S. W. J., Chao, G. T., \& Gardner, P. D. (1995). A longitudinal investigation of newcomer expectations, early socialization outcomes, and the moderating effects of role development factors. Journal of Applied Psychology, 80, 418-431.

Malik, A. R., Cooper-Thomas, H. D., \& Zikic, J. (2014). The neglected role of cultural intelligence in recent immigrant newcomers' socialization. International Journal of Cross Cultural Management, 14(2), 195-213.

Miller, V.D., \& Jablin, F. M. (1991). Information seeking during organizational entry: Influences, tactics, and a model of the process. Academy of Management Review, 16, 92-120.
Ng, V., Woo, S.E., Tay, L., \& Foster, J. (2016). Examining variability in values attributed to culture: Using personality as a relative benchmark. Journal of Cross-Cultural Psychology, 47, 981-996.

Podsiadlowski, A., Vauclair, M., Spieß, E., \& Stroppa, C. (2012). Social support on international assignments: The relevance of socioemotional support from locals. Journal of International Psychology. doi:10.1080/00207594.2012.669042.

Reif, J.A.M., \& Brodbeck, F.C. (2014). Initiation of negotiation and its role in negotiation research Foundations of a theoretical model. Organizational Psychology Review, 4, 363-381.

Reif, J. A. M., \& Brodbeck, F.C. (2017). When do people initiate a negotiation? The role of discrepancy, satisfaction, and ability beliefs. Negotiation and Conflict Management Research, 10, 46-66.

Richmond, A. (1993). Reactive migration: Sociological perspectives on refugee movements. Journal of Refugee Studies, 6, 7-24.

Ringeisen, T. (2013). Stressbewältigung im Kulturvergleich. In P. Genkova, T. Ringeisen \& F. T. L. Leong (Hrsg.), Handbuch Stress und Kultur (S. 255-278). Wiesbaden: Springer.

Ryan, L., Sales, R., Tilki, M., \& Siara, B. (2008). Social networks, social support and social capital: The experiences of recent Polish migrants in London. Sociology, 42(4), 672-690.

Safdar, S., Lay, C., \& Struthers, W. (2003). The process of acculturation and basic goals: Testing a multidimensional individual difference acculturation model with Iranian immigrants in Canada. Applied Psychology: An International Review, 52(4), 555-579.

Safdar, S., Struthers, W., \& Van Oudenhoven, J. P. (2009). Acculturation of Iranians in the United States, the United Kingdom, and the Netherlands: A test of the multidimensional individual difference acculturation (MIDA) model. Journal of Cross-Cultural Psycho$\log y, 40,468-491$.

Saks, A.M., \& Ashforth, B.E. (1997). Organizational socialization: making sense of the past and present as a prologue for the future. Journal of Vocational Behavior, 51, 234-279.

Saks, A. M., \& Ashforth, B.E. (2000). The role of dispositions, entry stressors, and behavioral plasticity theory in predicting newcomers' adjustment to work. Journal of Organizational Behavior, $21,43-62$.

Saks, A.M., \& Gruman, J.A. (2011). Organizational socialization and positive organizational behaviour: implications for theory, research, and practice. Canadian Journal of Administrative Sciences, 28, 14-26.

Saks, A.M., \& Gruman, J. A. (2014). Making organizations more effective through organizational socialization. Journal of Organizational Effectiveness: People and Performance, 1(3), 261-280.

Saks, A. M., Uggerslev, K.L., \& Fassina, N.E. (2007). Socialization tactics and newcomer adjustment: A meta-analytic review and test of a model. Journal of Vocational Behavior, 70, 413-446.

Sandhu, D., \& Asrabadi, B. (1994). Development of an acculturative stress scale for international students: Preliminary findings. Psychological Reports, 75, 435-448.

Schein, E. H. (1985). Organizational culture and leadership. San Francisco: Joessy-Bass.

Schwartz, S. H. (2011). Studying values: Personal adventure, future directions. Journal of Cross-Cultural Psychology, 41(2), 307-319.

Spieß, E., \& von Rosenstiel, L. (2010). Organisationspsychologie. München: Oldenbourg.

Stroppa, C., \& Spieß, E. (2011). The role of personal initiative on international assignments. International Journal of Intercultural Relations, 34, 234-245.

Taormina, R. J. (2009). Organizational socialization: the missing link between employee needs and organizational culture. Journal of Managerial Psychology, 24(7), 650-676.

Tornau, K., \& Frese, M. (2013). Construct clean-up in proactivity research: A meta-analysis on the nomological net of work-related proactivity concepts and their incremental validities. Applied Psychology: An International Review, 62, 44-96. 
Uslucan, H. (2013). Belastungen und Unterstützungsangebote von Migranten. In P. Genkova, T. Ringeisen \& F.T.L. Leong (Hrsg.), Handbuch Stress und Kultur (S. 381-396). Wiesbaden: Springer.

Ward, C. (1996). Acculturation. In D. Landis \& R. S. Bhagat (Hrsg.), Handbook of intercultural trainings (S. 124-147). Thousand Oaks CA: Sage.

Ward, C., \& Geeraert, N. (2016). Advancing acculturation theory and research: the acculturation process in its ecological context. Current Opinion in Psychology, 8, 98-104.

Ward, C., Bochner, S., \& Furnham, A. (2001). The psychology of culture shock. London: Routledge.

Wiesendahl, E. (2016). Der Kulturkonflikt um die Flüchtlingskrise und die politischen Folgen. Zeitschrift für Staats- und Europawissenschaften, 14(1), 53-79.

Wihler, A., Solga, M., \& Blickle, G. (2014). Personalentwicklung II: Karrieremanagement, Training und Beratung. In H. Schuler \& K. Moser (Hrsg.), Lehrbuch der Organisationspsychologie (S. 369-408). Bern: Huber.

Wood, R., \& Bandura, A. (1989). Impact of conceptions of ability on self-regulatory mechanisms and complex decision making. Journal of Personality and Social Psychology, 56, 407-415.

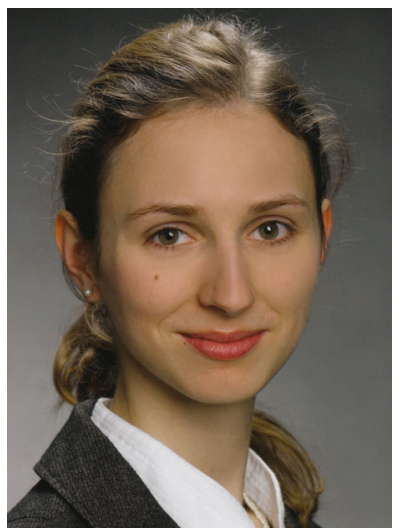

Dr. Julia A. M. Reif ist Mitarbeiterin am Lehrstuhl Wirtschaftsund Organisationspsychologie der Ludwig-Maximilians-Universität München. Sie forscht und publiziert zu den Themen Verhandlungen und Verhandlungsinitiierung, Team Prozesse, Beziehungsregulierung in ökonomischen Entscheidungssituationen, organisationale Akkulturation und Gesundheit in Organisationen.

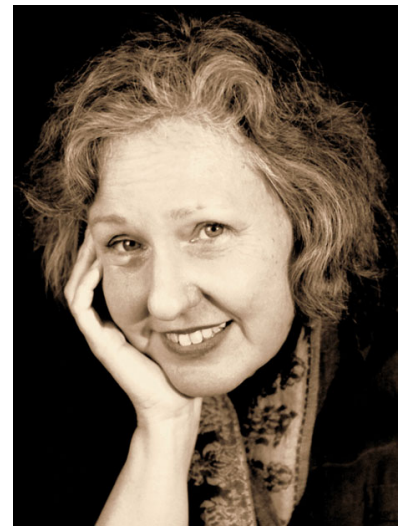

Apl. Prof. Dr. Erika Spieß is Mitarbeiterin am Lehrstuhl Wirtschafts- und Organisationspsychologie der Ludwig-Maximilians-Universität München. Sie forscht und publiziert vor allem zu den Themen Kooperation, interkulturelles Handeln in wirtschaftsnahen Kontexten und Gesundheit in Organisationen.

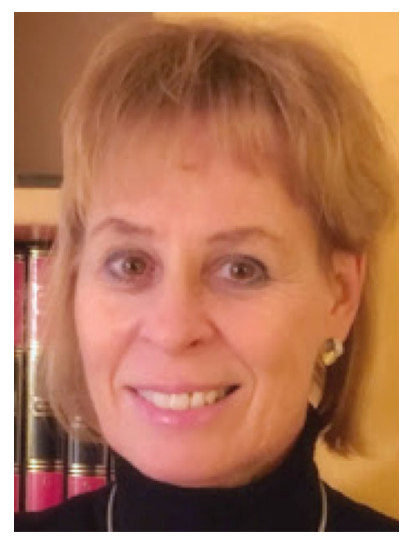

Prof. Dr. Rita Berger ist Professorin an der Fakultät für Psychologie der Universität Barcelona im Bereich Arbeits- und Organisationspsychologie. Sie forscht und publiziert vor allem zu den Themen Interkulturelle Führung, Teams und Gesundheit in Organisationen. 